Research Paper

\title{
Analysis of CDI37L and IL-I 7 Expression in Tumor Tissue as Prognostic Indicators for Gliblastoma
}

\author{
Xiangli Cui1, ${ }^{1, \#}$, Zhiqin $\mathrm{Xu}^{3 \#}$, Zhigang Zhao ${ }^{2}$, Dali Sui ${ }^{1}$, Xiaohui Ren${ }^{1}$, Qiming Huang4, Jiazhen Qin ${ }^{5}$, Limin \\ $\mathrm{Hao}^{4}$, Zhenguang Wang ${ }^{4}$, Li Shen ${ }^{6,7^{\bowtie}}$ and Song Lin ${ }^{\circledR}$ \\ 1. Department of Neurosurgery, Beijing Tiantan Hospital, Capital Medical University, Beijing, 100050, P.R. China; \\ 2. Department of Pharmacy, Beijing Tiantan Hospital, Capital Medical University, Beijing, 100050, P.R. China; \\ 3. Department of Neurosurgery, Peking Union Medical College Hospital, Chinese Academy of Medical Sciences, Beijing 100730, P.R. \\ China; \\ 4. Beijing Cellonis Biotechnologies Co., Ltd, 5 Kaituo Road, Zhongguancun Bio-Medicine Park, Beijing, 100085, P.R. China; \\ 5. The Affiliated Bayi Brain hospital, the Military General Hospital of Beijing PLA, 100700, P.R. China; \\ 6. The Department of Cell Biology, Peking University Health Science Center, Beijing,100091, P.R. China; \\ 7. Peking University Stem Cell Research Center, Beijing, 100191, P.R. China. \\ \# Common first author: Xiangli Cui, Zhiqin Xu.
}

$\bowtie$ Corresponding author: Song Lin or Li Shen, Song Lin, linsong2005@126.com, Tel:86-10- 010-67096509, Fax: 86-10- 010-67096856, Department of Neurosurgery, Beijing Tiantan Hospital, Capital Medical University, No.6 Tiantan Xili, Dongcheng district, Beijing, 100050, P. R. of China; Shen Li, shenli@bjmu.edu.cn, Tel:86-10-82802171, Fax: 86-10-82802171, Peking University Stem Cell Research Center, Xueyuan Road No.38, Haidian, Beijing, 100191, P. R. China.

(C) Ivyspring International Publisher. This is an open-access article distributed under the terms of the Creative Commons License (http://creativecommons.org/ licenses/by-nc-nd/3.0/). Reproduction is permitted for personal, noncommercial use, provided that the article is in whole, unmodified, and properly cited.

Received: 2012.10.29; Accepted: 2013.01.15; Published: 2013.01.16

\begin{abstract}
Glioblastoma multiforme (GBM) is the most common form of malignant glioma, characterized by genetic instability and unpredictable clinical behavior. GBM is marked by an extremely poor prognosis with median overall survival of 12 14 months. In this study, we detected the CDI37L-expressing cells and IL-17-expressing cells in tumor tissues resected from patients with GBM. Expression of CDI37L and IL-17 were assessed by immunohistochemistry, and the prognostic value of CDI37L and IL-I7 expression within the tumor tissues were assessed by Cox regression and Kaplan-Meier analysis. Immunohistochemical detection showed that positive cells of CDI37L and IL-I7 in glioblastoma tissue samples were $46.3 \%(I 9 / 4 I)$ and $73.2 \%(30 / 4 I)$ respectively. Expression of CDI37L was not correlated with overall survival of GBM patients $(P=0.594)$, while significantly longer survival rate was seen in patients with high expression of IL-17, compared to those with low expression of IL-I7 $(P=0.007)$. In addition, we also found that IL- I7 expression was significantly correlated with Progression-free survival (PFS) $(P=0.016)$ and death rate $(P=0.0 \mathrm{I})$. Furthermore, multivariate Cox proportional hazard analyses revealed that IL-I7 $(P=0.018)$ and PFS $(P=0.028)$ were independent factors affecting the overall survival probability. Kaplan-Meier analysis showed that PFS of high expression of IL- 17 group were significantly longer $(P=0.004)$ than low expression group with GBM. It is concluded that high levels of IL- 17 expression in the tumor tissues may be a good prognostic marker for patients with GBM.
\end{abstract}

Key words: CD137L, Interleukin-17, glioblastoma, overall survival.

\section{Introduction}

Glioblastoma is the most common and deadliest brain tumor, which escapes from immune recognition and kills most of the patients within one year of di- agnosis [1]. Glioblastomas, like other malignancies, are highly capable of overcoming host immune defenses through a variety of mechanisms, some of 
which are quite clear. Currently, there has been a growing interest in developing new methods of immunotherapy for treatment of glioblastoma, despite little is known about mechanisms of glioblastoma-specific immune responses [2]. A better understanding of the molecular interactions between the tumor and the host immune system may allow the development of novel integrated approaches based on the simultaneous control of tumor escape pathways and the activation of anti-tumor immune responses. Despite of the advances in surgery, chemotherapy, and radiation therapy, the prognosis for glioblastomas remains poor. Prognostic analysis for patients with GBM has not yet been fully studied. There is a correlation between the infiltration of immune cells within a tumor and the tumor development [3, 4]. Therefore, distribution of immune cells detected in brain glioma may provide useful information for the analysis of a patient's prognosis.

CD137L, a member of TNF superfamily, was found to be expressed following stimulation on professional APCs including DCs and macrophages as well as activated B cells and was also expressed on myeloid progenitors and hematopoietic stem cells CD137 ligand (CD137L)/CD137 interaction providing costimulatory signals to $\mathrm{T}$ cells independent of signaling pathways through the B7/CD28 interaction [5, 6]. CD137L can induce cytokine production and $\mathrm{T}$ cell proliferation in both the primary and secondary responses to Antigen, which promotes the development of CTL activity and anti-tumor immunity independent of signals through the CD28 molecule [7]. CD137L was found to be expressed in brain glioma in a modest percentage of patients. Although CD137L mRNA has been detected in patients with brain glioma [8-9], the distribution of CD137L-postive cells within the tumor and the relationship between CD137L and prognosis has not yet been investigated.

Recent studies[10-12] of autoimmune conditions in the central nervous system (CNS) have revealed that interleukin-17(IL-17) producing Type17 T-cells, specifically T-helper (Th)17 cells reactive to CNS autoantigens, manifest a highermigratory capability to the CNS parenchyma compared with other T-cell subpopulations due to their ability to penetrate the blood brain barrier (BBB). Interleukin-17 (IL-17) is a CD4 $\mathrm{T}$ cell-derived proinflammatory cytokine. IL-17 plays a positive role in the development of inflammation, GVHD and autoimmune diseases. An increase in the number of IL-17-postive cells in tumors has been detected in prostate, ovarian, and hepatocellular cancers [9].In this study, we examined the expression of CD137L and IL-17 in GBM by immunohistochemistry, and correlated these findings with available clinical data of patients with GBM. In addition, the expression of CD137 was assayed by flow cytometry, and its correlation with CD137L/CD137 expression was also analyzed with clinical classification of brain glioma. We evaluated the prognostic roles of CD137L and IL-17 in glioblastoma by Cox regression and Kaplan-Meier analysis.

\section{Material and Methods}

\section{Ethics statement}

All patients provided written informed consent for the current study and the clinical study was approved by the Medical Ethics Committee of Capital Medical University.

\section{Patients and tissue specimens}

Paraffin-embedded samples were obtained from 41 glioblastoma patients who underwent total or subtotal tumor resection in Beijing Tiantan Hospital during 2007 to 2011. Immunocompromised patients were excluded. None of the patients had received preoperative anticancer treatment. There were 18 male and 23 female patients with a median age of 47 years (range, 14-65 years). The follow-up data of the patients in this study are available and complete. The median follow-up for the entire cohort was 12.9 months (range, 4-24 months). All were stage IV cancer according to the TNM classification for brain cancer TNM staging system (International Union against Cancer, UICC). Each tumor was histologically graded, based on the WHO classification criteria. Overall survival (OS) was defined as the interval between the date of diagnosis and the date of death or the last known follow-up. All samples were coded anonymously in accordance with local ethical guidelines (as stipulated by the Declaration of Helsinki), and approved by the IRB (Internal Review Board) of Beijing tiantan hospital.

\section{Immunohistochemistry}

Formalin-fixed, paraffin-embedded samples were sectioned at $5 \mu \mathrm{m}$. Each tissue section was deparaffinized and rehydrated with graded ethanol. For antigen retrieval, the slides were boiled in EDTA (1 $\mathrm{mM} ; \mathrm{pH} \mathrm{8.0)}$ for $15 \mathrm{~min}$ in a microwave oven. Endogenous peroxidase activity was blocked with a $0.3 \%$ hydrogen peroxide solution for $10 \mathrm{~min}$ at room temperature. After rinsing with PBS, slides were incubated overnight at $4^{\circ} \mathrm{C}$ with respective primary antibodies which include goat anti-human CD137L polyclonal antibody (Santa Cruz Biotechnology; California, USA)(dilution 1/200) and mouse anti-human IL-17monoclonal antibody (Santa Cruz Biotechnolo- 
gy) (dilution 1/200). After three times of wash with PBS, sections were incubated with biotinylated anti-goat or anti- mouse secondary antibody (Zhongshan Golden Bridge Biotech, Beijing, China) respectively for $30 \mathrm{~min}$ at room temperature. Immunostaining was performed using the Envision System with diaminobenzidine (DakoCytomation, Glostrup, Denmark). Finally, the signal was developed with 3,3'-diaminobenzidine tetrahydrochloride (DAB), and all of the slides were counterstained with hematoxylin. Data were obtained by manually counting positively stained cells in five separate areas of slide regions with $200 \times$ high-power magnification. Mouse IgG1 (Santa) and normal goat IgG (Santa) were used as negative control stains.

\section{Statistical analysis}

Descriptive statistics were expressed as the mean $\pm \mathrm{SD}$ or median (range). The median value of immunohistochemically detected variables of CD137L and IL-17 was used as cut-off in our results. Chi-squared test or Fisher exact test was used to assess the relationship between the expression of CD137L or IL-17 and the clinic pathological features. Correlations between CD137L and IL-17 were evaluated by the Pearson correlation coefficient. Prognostic factors were evaluated by bivariable analyses using log rank test of Kaplan-Meier methodology and by multivariable analyses using Cox proportional haz-

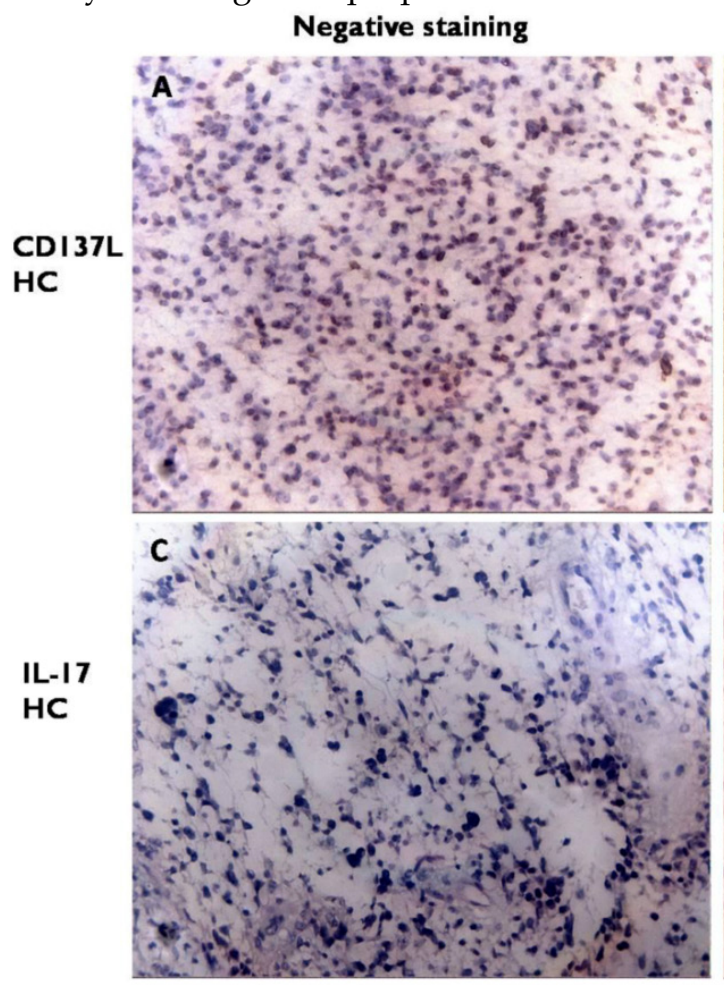

ards model. A two-sided $P$ value $<0.05$ was considered statistically significant. All statistical analyses were performed with SPSS software (version 16.0; SPSS Inc., Chicago, IL, USA).

\section{Results}

\section{Study population}

The patient demographics are presented in Table 1. Of the 41 patients examined, 15 (36.6\%) died before the end of the observation. The median age of the population studied was 47 years (range 14-67 years). Overall, All patients presented with WHO grade IV and glioblastoma. The median follow-up for the entire cohort was 12.9 months (range 4-24 months). The 2 -year survival incidence for the entire study population was $63.4 \%$.

\section{Immunohistochemical staining}

The occurrence of CD137L and IL-17 high-expressed cells were $46.3 \%$ (19 of 41 ) and $73.2 \%$ (30 of 41) respectively. Both CD137L and IL-17 positive staining were mostly localized to the cytoplasm. IL-17 may be situated in the cytoplasm but CD137L should be on the surface to be functional (Figure 1 and Table 2). Less than $15 \%$ tumor cells expressing CD137L or IL-17 means low expression. More than $15 \%$ tumor cells expressing CD137L or IL-17 in glioma tissue means high expression.

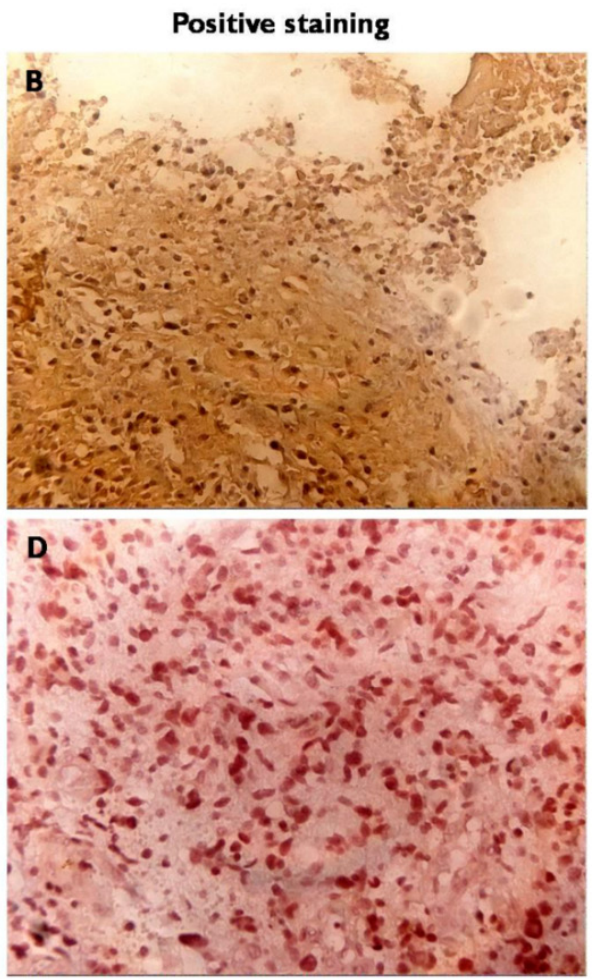

Figure I. Expression of CDI37L and IL-I7 in tumor. Consecutive sections were used for immunohistochemical analysis of CDI37L (A, B) and IL- 7 expression (C, D) (A, C negative expression (200×magnification); B, D positive expression (200×magnification)). 
Table I. Clinical characteristic of $4 \mid$ patients with brain glioblastoma.

\begin{tabular}{ll}
\hline Groups & Characteristics \\
\hline Age, years & Years \\
median & 47 \\
range & $14-67$ \\
Gender & Number $\%$ \\
male & $18(43.90)$ \\
female & $23(56.10)$ \\
Karnofsky, KPS & Score \\
median & 80 \\
range & $30-90$ \\
Tumor (T) stage & Number \\
T4 & 41 \\
Progression-free survival, PFS & Months \\
median & 9.84 \\
range & $3-19$ \\
Death & Number $\%$ \\
no & $26(63.41)$ \\
yes & $15(36.59)$ \\
Histologic grade & Number \\
IV & 41 \\
Recurrence & Number\% \\
no & $39(95.12)$ \\
yes & $2(4.88)$ \\
\hline
\end{tabular}

Table 2. Descriptive statistics of immunohistochemical variables.

\begin{tabular}{llll}
\hline Variables & Low-expressed & High-expressed & $\begin{array}{l}\text { Positive } \\
\text { rate }\end{array}$ \\
\hline $\begin{array}{l}\text { CD137L-producing } \\
\text { cells in tumor }\end{array}$ & 22 & 19 & $46.34 \%$ \\
$\begin{array}{l}\text { IL-17-producing cells } \\
\text { in tumor }\end{array}$ & 30 & $73.17 \%$ \\
\hline
\end{tabular}

\section{Relationship between the expression of CDI37L and IL-I 7 in tumor and pathological features of patients with glioblastoma}

IL-17 expression significantly correlated with progression-free survival (PFS) $(P=0.029)$ and death rate $(P=0.01)$ (Table 3$)$. IL-17 expression did not significantly $(p>0.05)$ correlated with age, gender, or Karnofsky (KPS) in patients with glioblastoma (Table $3)$. CD137L expression did not significantly $(p>0.05)$ correlate with age, gender, Karnofsky (KPS), PFS, as well as death rate in patients with glioblastoma (Table 3). But Kaplan-Meier PFS curves for high expression of IL-17 group were significantly different from low IL-17 expression group $(P=0.004)$ (Figure 4$)$. However, CD137L expression did not significantly $(P>0.05)$ correlate with age, gender, Karnofsky (KPS), PFS, or death in patients with glioblastoma (Table 3).

\section{Univariate and multivariate analysis of prog- nostic variables in patients with glioblastoma}

To identify the variables with potential prognostic significance in patients with glioblastoma, univariate and multivariate analyses using log rank test of Kaplan-Meier methodology and Cox proportional hazard model were performed. These analyses assessed the potential prognostic significance of CD137L and IL-17 expressions and other clinical pathological parameters for patients with glioblastoma. Univariate analysis showed that expression of IL-17 and PFS were both significant prognostic factors (Table 4). Multivariate analysis revealed that IL-17 expression was an independent predictor of survival $(P=0.018)$, as was PFS $(P=0.028)$. The relative risk in patients with low levels of IL-17 was $0.072(0.07)$ times greater than that in patients with higher expression of IL-17 (Table 4). However, CD137L expression did not associate with overall survival $(p>0.05)$ by univariate and multivariate analysis, neither did age, gender, and KPS $(p>0.05)$.

\section{Correlation between expressions of CDI37L and IL-I 7 in tumor and overall survival}

The prognostic value of CD137L and IL-17 expression on PFS and overall survival of GBM was evaluated. Using a Kaplan-Meier curve assessment, we found that expression of CD137L was not an independent predictor for prognosis of patients with GBM. The two-year overall survival probability among patients with high levels of CD137L was not significantly higher than those with low levels expression $(P=0.594)$ (Figure 2). However, patients expressing high levels of IL-17 had a better two-year overall survival probability than those expressing low levels $(P=0.007)$ (Figure 3). Therefore, we found that negative or low expression of IL-17 in was an independent predictor of poor prognosis in patients with glioblastoma. High expression of IL-17 is a indicator of longer overall survival in patients with glioblastoma. In this study, we found there was no significant correlation between the expression level of CD137L and IL-17 in the tumor tissue. 
Table 3. Correlations between CDI37L, IL-I7 expression in tumor tissues and clinic pathological features of patients with brain glioblastoma

\begin{tabular}{|c|c|c|c|c|c|c|}
\hline \multirow[t]{2}{*}{ Variables } & \multicolumn{2}{|c|}{ CD137L-positive cells } & \multirow[t]{2}{*}{$P$ value } & \multicolumn{2}{|c|}{ IL17-positive cells } & \multirow[t]{2}{*}{$P$ value } \\
\hline & negative & positive & & Low & High & \\
\hline \multicolumn{7}{|l|}{ Age, years } \\
\hline$<60$ & 16 & 15 & & 20 & 11 & \\
\hline$\geq 60$ & 6 & 4 & 0.80 & 5 & 5 & 0.43 \\
\hline \multicolumn{7}{|l|}{ Gender } \\
\hline male & 11 & 9 & & 12 & 6 & \\
\hline female & 11 & 12 & 0.71 & 13 & 10 & 0.52 \\
\hline \multicolumn{7}{|c|}{ Karnofsky, KPS } \\
\hline$\leq 60$ & 5 & 2 & & 6 & 1 & \\
\hline$>60$ & 17 & 17 & 0.64 & 19 & 15 & 0.15 \\
\hline \multicolumn{7}{|c|}{ Progression-free survival, PFS, months } \\
\hline$<12$ & 11 & 13 & & 18 & 6 & \\
\hline$\geq 12$ & 11 & 6 & 0.94 & 7 & 10 & $0.02^{\mathrm{a}}$ \\
\hline \multicolumn{7}{|l|}{ Death } \\
\hline no & 13 & 13 & & 12 & 14 & \\
\hline yes & 9 & 6 & 0.74 & 13 & 2 & $0.01^{\mathrm{a}}$ \\
\hline
\end{tabular}

Chi-squared tests, aP<0.05.

Table 4 Univariate and Multivariat analyses of variables associated with survival

\begin{tabular}{lcccccc}
\hline Variables & \multicolumn{3}{c}{ Univariate analysis } & \multicolumn{3}{c}{ Multivariate analysis } \\
\hline & Log-Rank test & $95 \%$ CI & $P$ value & HR & $95 \%$ CI & $P$ value \\
\hline CD137L-positive cells(Low vs High) & 0.29 & $12.52-19.48$ & 0.59 & 0.75 & $0.27-2.14$ & 0.60 \\
IL17-positive cells(Low vs High) & 7.28 & $19.86-24.51$ & $0.01^{\text {a }}$ & 6.24 & $1.37-28.46$ & 0.02 a \\
Age, years $(\geq 60$ vs $<60)$ & 0.00 & $11.41-17.37$ & 0.96 & 0.72 & $0.23-2.32$ & 0.58 \\
Gender(male vs female) & 0.09 & $14.94-21.99$ & 0.76 & 1.39 & $0.48-4.00$ & 0.54 \\
KPS( $\leq 60 v s>60)$ & 0.01 & $12.87-20.63$ & 0.94 & 1.59 & $0.43-5.88$ & 0.48 \\
PFS, months $(\geq 12$ vs $<12)$ & 6.01 & $12.78-18.76$ & $0.01^{\text {a }}$ & 0.07 & $0.01-0.75$ & $0.03^{\text {a }}$ \\
\hline
\end{tabular}

Fisher's Exact, a $<<0.05 ; V$ means vs.; KPS: Karnofsky; PFS: Progression-free survival.

HR Hazard Ratio, CI confidence interval

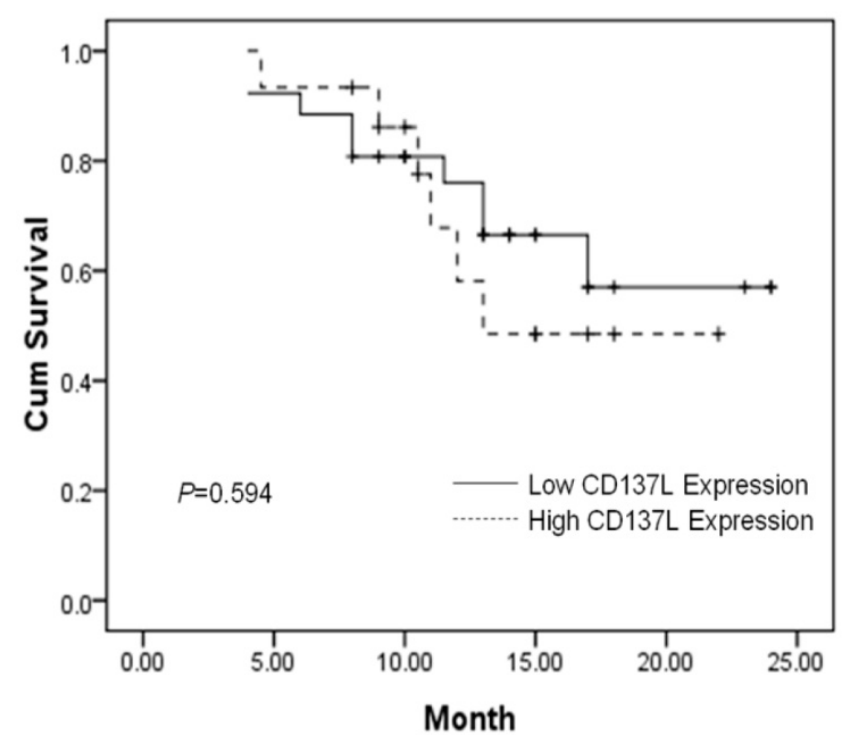

Figure 2. Patients expressing high CDI37L in tumor tissues do not show significantly improved two-year overall survival $(P=\mathbf{0 . 5 9 4})$. Survival curves of $4 \mathrm{I}$ brain glioblastoma patients with different CDI37L expression are shown. Kaplan-Meier survival curves for positive expression of CDI37L group were not significantly different ( $P=0.594$, log-rank test) from negative CDI37L expression group in tumor tissues of $4 \mathrm{l}$ brain glioblastoma patients. 


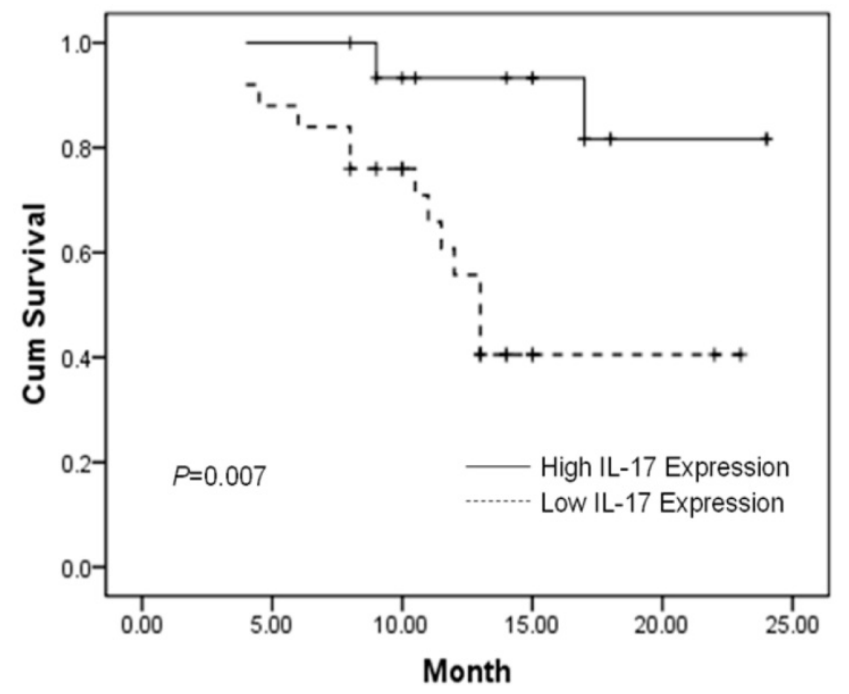

Figure 3. Patients expressing high IL- $I 7$ in tumor tissues show significantly better two-year overall survival $(P=0.007)$. Survival curves of $4 \mid$ brain glioblastoma patients with different IL-I7 expression are shown. Kaplan-Meier survival curves for high expression of IL- 17 group were significantly different ( $P=0.007$, log-rank test) from low IL- I 7 expression group in tumor tissues of $4 \mathrm{I}$ brain glioblastoma patients.

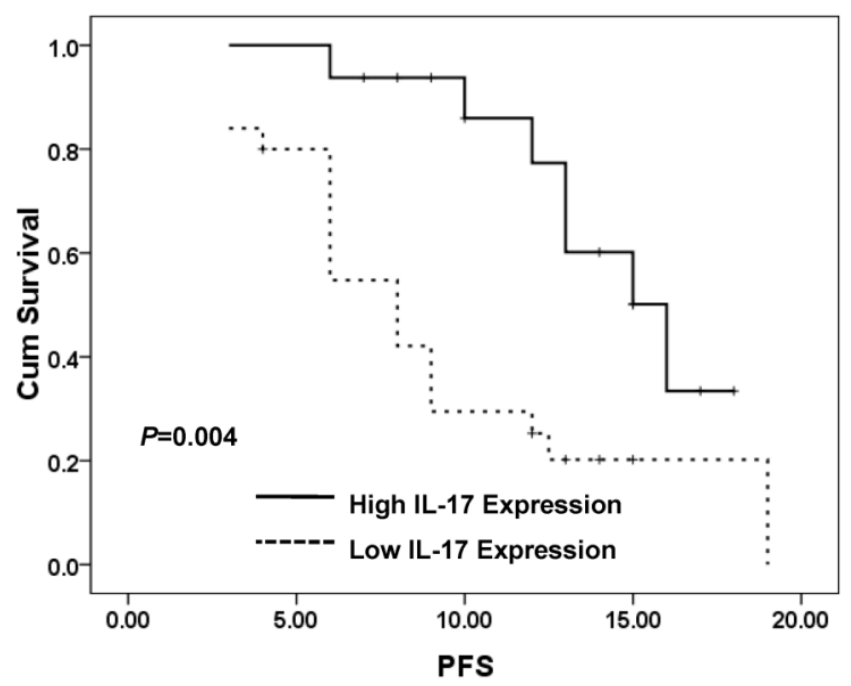

Figure 4. Patients expressing high IL-I 7 in tumor tissues show significantly better Progress-free survival than low-expression of IL-I7(PFS) $(\boldsymbol{P}=\mathbf{0 . 0 0 4})$. PFS curves of 41 brain glioblastoma patients with different IL-I7 expression are shown. Kaplan-Meier PFS curves for high expression of IL-I7 group were significantly different ( $P=0.004$, log-rank test) from low IL- 17 expression group in tumor tissues of $4 \mathrm{I}$ brain glioblastoma patients.

\section{Discussion}

Glioblastoma is one of the most malignant brain tumor associated with the poor 5-year survival rates among all human cancers [13-14]. The median overall survival was about 12-14 months [15]. Therefore, identifying molecular targets that could provide prognostic information is greatly needed and would be helpful for its therapy. CD137L, the counterreceptor for CD137(4-1BB), is a member of the TNF (ligand) superfamily and serves as a secondary signal to activated $T$ cells. CD137 is an inducible member of the TNFR superfamily that can induce cytokine production, expansion, and functional maturation of T cells, NK cells, monocytes, and dendritic cells [16]. After prolonged TCR/CD28 activation in vitro, T cells increase CD137 expression and upon binding become strongly adherent to fibronectin. With regard to tumor biology, binding of CD137 has been demonstrated to prevent and even rescue anergic CD8 T cells in a number of tolerance-inducing models [17]. CD137 agonist antibody has also been shown to overcome immunological ignorance (where CTL were not deleted or anergized, but simply not activated), allowing immunization with tumor-derived peptide to induce a protective CTL response. Engagement of CD137 by CD137L on antigen-presenting cells may lead to the generation of cytolytic $T$ cells as well as promotion of T-helper 1 cells, which produce high levels of IFN- $\gamma$ [18]. Most cell lines express CD137L, but none expresses B7-1. Anderson and his colleague had reported that high expressing CD137 glioma cells were resistant to vincristine (VCR), and CD137L expression was correlated with survival and age rather than pathological grade of glioma [11]. Mu and his colleague had reported that patients with high expression of CD137L had a longer overall survival) [12], but the result of our study (Fig.2) was opposite to that of those studies. We consider that such difference is due to the fact that those studies checked surface expression of CD137L, not intratumoral expression of CD137L as we did.

IL-17 has been of great interest recently owing to the discovery that the production of IL-17 characterizes Th17 cells. The development of Th17 cells is distinct from the development of Th1, Th2 and regulatory $\mathrm{T}$ (Treg) cells and is characterized by predominant production of IL-17 as well as their developmental control by retinoic acid-related orphan receptor (ROR) $\gamma t$ and signal transducer and activator of transcription 3 (STAT3) [19]. Th17 cells produce IL-17, IL-6, IL-21, IL-22, IL-23 and TNF-a. Although publications on the role of IL-17 and IL-17-associated cytokines in cancer to date report both pro- and anti-tumor functions, it appears consistent that adoptively transferred Type17 T-cells may mediate potent anti-tumor immunity due to their longevity as well as ability to develop into Type1 cells in cancerbearing hosts. An increase of IL-17-positive cells has been ob- 
served in prostate and hepatocellular cancers [20]. Patient's survival in ovarian cancer and small cell lung cancer positively correlated with IL-17 expression [21]. In our study, we found that patients with high level expression of IL-17 in tumor showed significantly longer overall survival than those with low level experession of IL-17 ( $\mathrm{P}=0.007$, Figure 3). A recent study showed that expression of IL-17 is detected intratumorally and in an effector T-cell subset that expresses tumor antigen-specific CD4 [22]. CD137L is expressed in many cells including dendritic cells that can modulate the proliferation of effector $\mathrm{T}$ cells and play a potential role in anti-tumor immunity [23, 24]. In this study, we detected the expression of CD137L and IL-17 within the brain glioblastomas and assessed their relationship with patients' prognosis. This is the first study to show that CD137L and IL-17 may serve as prognostic indicators for glioblastoma. We used immunohistochemistry to characterize CD137L and IL-17 positive cells in tumor tissues of patients with glioblastoma after resection and analyzed the association between the prognosis and the expression of CD137L and IL-17.

In this study, we collected 41 glioblastoma samples among which 46.3\% (19/41) expressed CD137L in the cytoplasm. CD137L expressed on glioma cells could function as a co-stimulatory molecule of $\mathrm{T}$ cell activation for the production of cytokines, most notably interferon-gamma (IFN- $\gamma)$, in co-culture of T cells and glioma cells. Moreover, IL-17 was extensively expressed in the cytoplasm. We further analyzed the relationship between expression of CD137L or IL-17 and the clinic pathologic features of glioblastoma. CD137L expression did not significantly correlate with age and gender. There was no significant association between the expression of CD137L and other clinical and pathological features. Of note, we found a statistically significant correlation between the expression of IL-17 and PFS ( $\mathrm{P}=0.016)$ and death rate $(\mathrm{P}=0.01)$. Some other studies have shown that IL-17 expression and the presence of Th17 cells were associated with clinical stage in glioma tissue $[25,26]$. The mRNA expression of IL-17 in glioma may influence the progression of glioma. Kaplan-Meier survival analysis showed that the two-years overall survival rate of patients with high levels expression of IL-17 was significantly higher than those with low levels of IL-17 expression $(\mathrm{P}=0.007)$. This is consistent with a recent report on human ovarian cancer [9]. However, other studies observed that IL-17 increased intratumorally in glioma. IL-17 positive cells may play an important role in glioma tumorigenesis and progression because Th17 polarization may be induced by regulatory T cells [25, 26]. Different types of TNM staging of glioma and immunological status may contribute to different results. While there was no significant correlation between the level of CD137L and patient survival $(\mathrm{P}=0.594)$ in our study, CD137L-positive cells were found to correlate with tumor progression [11]. On the other hand, some studies have found that the ligation of CD137L on tumor cells with CD137 on T cells induced IFN- $\gamma$ production by $\mathrm{T}$ cells. This triggered tumor cells to produce IL-8 that regulating tumor progression [27]. These results are conflicting and require further investigation.

By using univariate and multivariate analyses, we found that high expression of IL-17 was significant prognostic factor for glioblastoma. Multivariate analysis revealed that IL-17 expression was an independent predictor for survival $(\mathrm{P}=0.018)$ and PFS $(\mathrm{P}=0.028)$. IL-17 expression may also be a potential prognostic factor in all patients with glioblastoma. However, we found no significant correlation between the expression of CD137L and the independent predictor. Overall, our data show that both CD137L and IL-17-expressing cells are generally expressed in brain glioblastoma. Though we found that CD137L expression is not significantly correlated with overall survival and does not serve as an independent prognostic indicator in glioblastoma, our results suggested that IL-17 expression is significantly correlated with PFS $(\mathrm{P}=0.016)$ and death rate $(\mathrm{P}=0.01)$. Meanwhile, multivariate Cox proportional hazard analyses revealed that IL-17 $(\mathrm{P}=0.018)$ and PFS $(\mathrm{P}=0.028)$ are independent factors affecting the two-year overall survival probability. Kaplan-Meier PFS curves also showed that high expression of IL-17 was significantly correlated with PFS ( $\mathrm{P}=0.004$, log-rank test). Our conclusion is that the expression levels of intratumoral IL-17 can be an independent prognostic indicator for patients with glioblastoma. The molecular mechanism for this correlation remains unknown. Relevant studies may help better understand the role of IL-17 in the development of glioblastoma.

\section{Acknowledgments}

This work was supported by Han Xiao and Chunyu Qi.

\section{Financial support}

This work was supported by Beijing Natural Science Foundation (7122061).

\section{Competing Interests}

The authors declare that there are no conflicts of interest in this study. No external funding, apart from the support of the authors' institution, was available 


\section{for this study.}

\section{References}

1. Huse JT, Holland EC. Targeting brain cancer: advances in the molecular pathology of malignant glioma and medulloblastoma. Nat Rev Cancer. 2010; 10(5):319-331.

2. Stupp R, Mason WP, van den Bent MJ, et al. Radiotherapy plus concomitant and adjuvant temozolomide for glioblastoma. N. Engl. J. Med. 2005; 352 (10):987-996.

3. Liu S, Lachapelle J, Leung S, Gao D, Foulkes WD, Nielsen TO. CD8+ lymphocyte infiltration is an independent favorable prognostic indicator in basal-like breast cancer. Breast Cancer Res. 2012; 14(2):R48.

4. Deschoolmeester V, Baay M, Van Marck E, Weyler J, Vermeulen P, Lardon F, Vermorken JB. Tumor infiltrating lymphocytes: an intriguing player in the survival of colorectal cancer patients. BMC Immunol. 2010; 11:19.

5. DeBenedette MA, Shahinian A, Mak TW, et al. Costimulation of CD28- T lymphocytes by 4-1BB ligand. J Immunol. 1997; 158(2):551-559.

6. Cannons JL, Lau P, Ghumman B, et al. 4-1BB ligand induces cell division, sustains survival, and enhances effector function of CD4 and CD8 T cells with similar efficacy. J Immunol. 2001; 167(3):1313-1324.

7. Lynch DH. The promise of 4-1BB (CD137)-mediated immunomodulation and the immunotherapy of cancer. Immunol Rev. 2008; 222:277-286.

8. Mou YG, Xie H T, Peng H, et al. Clinical significance of 4-1BBL protein expression in human glioma tissues. Chin J Pathophysiol. 2010; 26(12):2342-2346.

9. Weaver CT, Hatton RD, Mangan PR, et al. IL-17 family cytokines and the expanding diversity of effector T cell lineages. Annu Rev Immunol. 2007; 25:821-852

10. Kryczek I, Banerjee M, Cheng P, et al. Phenotype, distribution, generation, and functional and clinical relevance of Th17 cells in the human tumour environments. Blood 2009; 114:1141-1149.

11. Anderson RC, Anderson DE, Elder JB, et al. Lack of B7 expression, not human leukocyte antigen expression, facilitates immune evasion by human malignant gliomas. Neurosurgery. 2007; 60(6):1129-1136.

12. Mu YG, Peng H, Zhang JY, et al. Expression of costimulator 4-1BBL and B7-1 on glioma cell lines. Ai Zheng. 2006; 25(3):326-329.

13. Fleming AJ, Chi SN. Brain tumors in children. Curr Probl Pediatr Adolesc Health Care. 2012; 42(4):80-103

14. Krex D, Klink B, Hartmann C, et al. Long-term survival with glioblastoma multiforme. Brain. 2007; 130(Pt 10): 2596-2606.

15. Ohgaki H, Dessen P, Jourde B, et al. Genetic pathways to glioblastoma: a population-based study. Cancer Res 2004; 64(19):6892-6899.

16. Wilcox, R. A., K. Tamada, S. Strome, and L. Chen. Signaling through NK cell-associated CD137 promotes both helper function for CD8 cytolytic T cells and responsiveness to IL-2 but not cytolytic activity. J. Immunol. 2002;169:4230-4236

17. Wilcox, R. A., K. Tamada, D. B. Flies, G. Zhu, A. I. Chapoval, B. R. Blazar,W. M. Kast, and L. Chen. Ligation of CD137 receptor prevents and reversesestablished anergy of CD8 cytolytic T lymphocytes in vivo. Blood.2004;103: 177-184.

18. Kim, Y-J, Kim, S H, Mantel, P, et al. Human 4-1BB regulates CD28 co-stimulation to promote Th1 cell responses. Eur J Immunol. 1998; 28(3): 881-890.

19. Zhang Q, Liu S, Ge D, et al. Interleukin-17 Promotes Formation and Growth of Prostate Adenocarcinoma in Mouse Models. Cancer Res. 2012; 72(10):2589-2599.

20. Kuang DM, Peng C, Zhao Q, et al. Tumor-activated monocytes promote expansion of IL-17-producing CD8+ T cells in hepatocellular carcinoma patients. J Immunol. 2010; 185(3):1544-1549.

21. Gu FM, Gao Q, Shi GM, et al. Intratumoral IL-17(+) Cells and Neutrophils show Strong Prognostic Significance in Intrahepatic Cholangiocarcinoma. Ann Surg Oncol. 2012; [Epub ahead of print]

22. Hamaï A, Pignon P, Raimbaud I, et al. Human TH17 Immune cells specific for the tumor antigen MAGE-A3 convert to IFN- $\gamma$-secreting cells as they differentiate into effector T cells in vivo. Cancer Res. 2012; 72(5):1059-1063.

23. Sharma RK, Schabowsky RH, Srivastava AK, et al. 4-1BB ligand as an effective multifunctional immunomodulator and antigen delivery vehicle for the development of therapeutic cancer vaccines. Cancer Res. 2010; 70(10):3945-3954.

24. Wu C, Guo H, Wang Y, Gao Y, Zhu Z, Du Z. Extracellular domain of human 4-1BBL enhanced the function of cytotoxic T-lymphocyte induced by dendritic cell. Cell Immunol. 2011; 271(1):118-123.
25. Wainwright DA, Sengupta S, Han Y, et al. The presence of IL-17A and T helper 17 cells in experimental mouse brain tumors and human glioma. PLoS One. 2010; 5(10):e15390.

26. Hu J, Mao Y, Li M, Lu Y. The profile of Th17 subset in glioma. Int Immunopharmacol. 2011; 11(9):1173-1179.

27. Wang Q, Zhang P, Zhang Q, et al. Analysis of CD137 and CD137L expression in human primary tumor tissues. Croat Med J. 2008; 49(2):192-200. 